\title{
Hyperkalemia Secondary to Prophylactic Heparin Use in a Trauma Patient: Case Report
}

\author{
Marco Custodio, PharmD', Errington C. Thompson, MD²
}

ABSTRACT

This case report describes a complex trauma patient who develops hyperkalemia secondary to heparin. Heparin is a commonly used drug in trauma patients. Physicians need to be aware of this potentially harmful adverse reaction in these high-risk patients.

\section{KEYWORDS}

Trauma, Heparin, Anticoagulation, Hyperkalemia, Critical Care, Renal Insufficiency
Author affiliations are listed at the end of this article.

Correspondence to: Errington C. Thompson, MD Marshall University Joan C. Edwards School of Medicine thompsoner@marshall.edu

\section{INTRODUCTION}

Deep venous thrombosis (DVT) is a serious potential complication of trauma. In his classic article, Geerts et al identified risk factors for developing DVT in trauma patients, with head injuries, spinal cord injuries, and lower extremity fractures at the highest risk. ${ }^{1}$ Prophylactic anticoagulation is recommended in these high-risk patients. ${ }^{2-4}$ We present a high-risk trauma patient who developed a rare complication of hyperkalemia secondary to heparin.

\section{CASE PRESENTATION}

A 47-year-old male was an unrestrained passenger involved in a rollover motor vehicle crash. The patient was confused at the scene. Emergency medical service transported the patient to Cabell Huntington Hospital. The patient arrived hemodynamically stable (blood pressure 124/106 mm Hg, pulse 76 beats/ minute, respiratory rate 20 respirations/minute, oral temperature $\left.98.1^{\circ} \mathrm{F}\right)$. The patient was not moving his legs, and he underwent multiple computed tomography (CT) scans - head, cervical spine, chest, abdominal, and pelvis with reconstructions of the thoracic and lumbar spines. No intracranial injury was identified. The $\mathrm{CT}$ of the cervical spine revealed bilateral jumped facets at C7-T1 with high-grade spinal stenosis with possible crush injury to the spinal cord. A fracture of the sternum was seen on the patient's chest CT along with a posterior mediastinal paraspinous hematoma which extended from T1 to T4. The CT of the abdomen/pelvis revealed an acetabular fracture as well as an $\mathrm{L} 5$ superior endplate fracture without compromise of the spinal canal. The kidneys were normal. Admission labs were unremarkable including a potassium level of $3.8 \mathrm{mEq} / \mathrm{L}$. The patient's past medical history included hypertension and diabetes. The patient also reportedly had a stroke several years earlier and was taking aspirin prophylactically. The patient was a one pack per day smoker.

While in the emergency room, the patient developed neurogenic shock but responded appropriately to resuscitative efforts which included fluid and norepinephrine. He was admitted to the intensive care unit. Orthopedics and neurosurgery were consulted. The patient was placed in traction via a distal femur pin for his acetabular fracture. The patient was taken to the operating room by neurosurgery on post-injury day 1 where he 


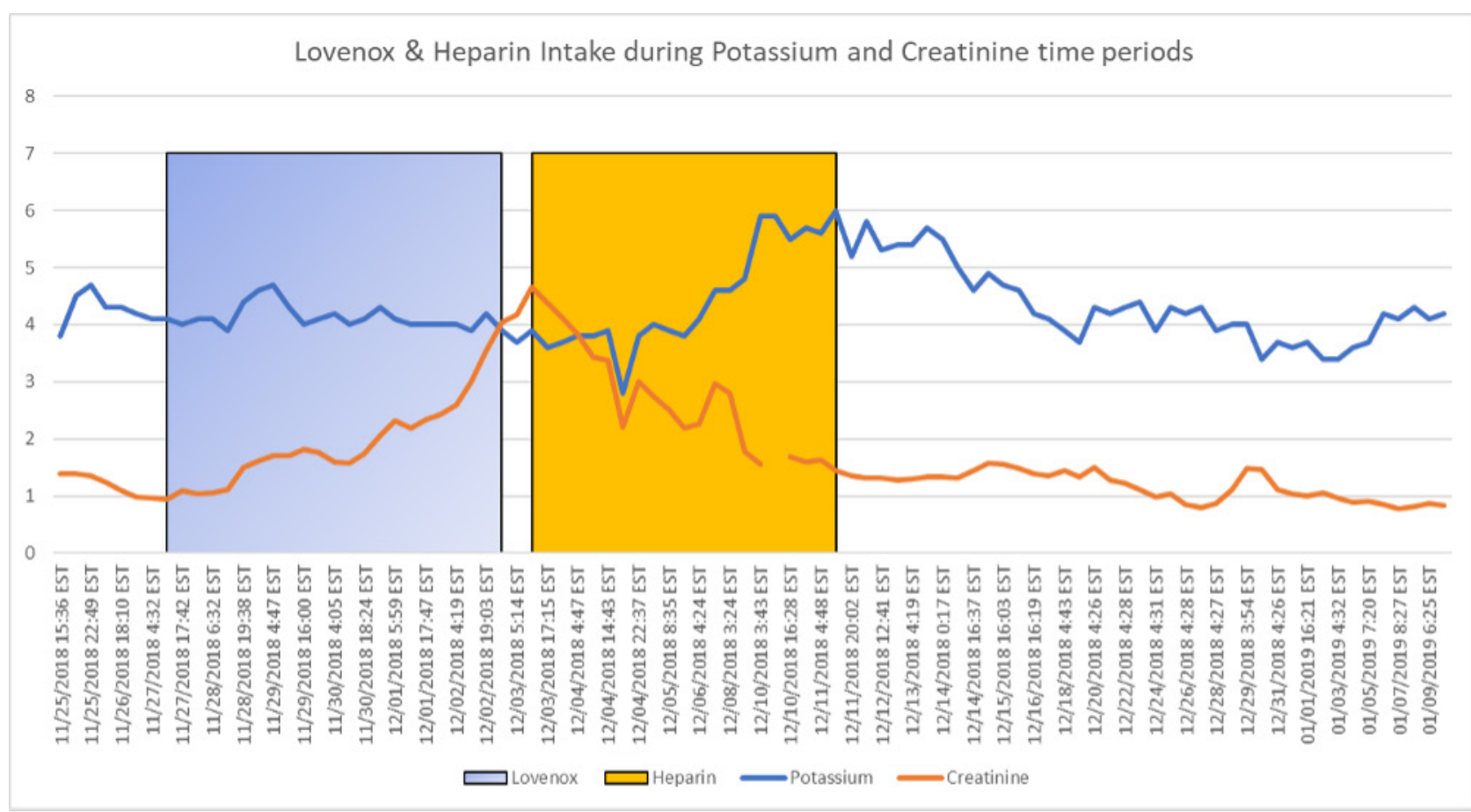

FIGURE 1. The blue line is the patient's potassium level. The orange line is the serum creatinine. The blue shade box represents when the patient was on Lovenox and the orange box is when the patient was on heparin.

underwent reduction of his C7/T1 jumped facets with internal fixation. The patient remained intubated postoperatively.

The patient spiked a fever on post-injury day 2 . He was pan-cultured which revealed communityacquired pneumonia with Haemophilus influenza. He was treated with broad-spectrum antibiotics and subsequent, directed de-escalation based on susceptibility results. However, the patient developed septic shock in spite of adequate antibiotic coverage for $\mathrm{H}$. influenza. He stabilized within hours after receiving a combination of fluids and pressors.

Also, on post-injury day 2 , the patient's creatinine was within normal limits $(0.98 \mathrm{mg} / \mathrm{dL})$ and he was started on prophylactic enoxaparin as per the hospital protocol for patients with spinal cord injuries. However, his renal function deteriorated, and his creatinine was elevated to $2.32 \mathrm{mg} / \mathrm{dL}$ on post-injury day 6 . In spite of adequate blood pressure and no evidence of hypovolemia, the patient's creatinine continued to climb to $2.60 \mathrm{mg} / \mathrm{dl}$. Nephrology was consulted and a retroperitoneal ultrasound was performed, which was normal. The patient was switched from D51/2 NS to D5W infused at $75 \mathrm{~mL} /$ $\mathrm{hr}$. The cause of his worsening renal function was thought to be multifactorial relative to shock and CT contrast dye. The patient was switched from enoxaparin to 5,000 units of subcutaneous heparin three times daily.

Simultaneous to the patient's decline in renal function, the patient's respiratory status continued to deteriorate. Using a lung-protective strategy to manage the ventilator, the patient was placed on airway pressure release ventilation (APRV). However, the patient developed progressive hypoxia in spite of being on mechanical ventilation. The patient was pan-cultured for suspected sepsis; all cultures were negative. The patient became more hypoxic with tachycardia and hypotension. The patient was not responsive to fluid challenges and norepinephrine was started to support the patient's blood pressure. Our goal was to maintain a mean arterial pressure greater than $65 \mathrm{mmHg}$. The patient was started on broad-spectrum antibiotics of piperacillintazobactam and linezolid.

On post-injury day 10 , a noninvasive cardiac monitor 
was placed on the patient and an echocardiogram was obtained which revealed an ejection fraction of $60 \%$ with no wall motion abnormalities. However, the patient's inferior vena cava was dilated with poor inspiratory collapse. The non-invasive cardiac monitor revealed a low cardiac index and a modestly high systemic vascular resistance which suggested that the patient's hypotension was probably secondary to cardiac dysfunction. The patient became persistently tachycardiac while on norepinephrine. He was switched to dobutamine which resolved his tachycardia and increased his mean arterial pressure to greater than $65 \mathrm{mmHg}$.

The patient's respiratory status began to improve, and the patient's dobutamine was weaned off. The patient was fluid overloaded. Furosemide was given and the patient voided more than six liters of fluid over two days. The patient's potassium climbed to $6 \mathrm{mEq} / \mathrm{L}$ in spite of conservative measures such as removing potassium from IV fluids, so insulin and albuterol were given. Nephrology suggested that the patient's subcutaneous heparin be stopped. The heparin was discontinued and fondaparinux was started at $2.5 \mathrm{mg}$ subcutaneously (Figure 1). Over the next ten days, the patient continued to slowly improve and was switched back to conventional ventilation. His potassium began to decrease and by post-injury day 25 , it was within the normal range. The patient did not require dialysis. The patient underwent a tracheostomy and percutaneously placed gastrostomy tube without difficulty. The patient was extubated without problems; he was alert and interactive. Prior to discharge, he was tolerating a regular diet and was transferred to a rehabilitation center on post-injury day 46.

\section{DISCUSSION}

In this case report, we present a complex trauma patient who was involved in a motor vehicle crash. The patient sustained multiple injuries including a C7 spinal cord injury, fractured sternum, posterior mediastinal paraspinous hematoma which extended from $\mathrm{T} 1$ to T4, an acetabular fracture, and an L5 superior endplate fracture without compromise of the spinal canal. In the ER, he developed neurogenic shock and required pressors. The patient's respiratory status and renal function declined early during his hospital course. He was originally placed on enoxaparin for DVT prophylaxis but was switched to heparin because of his decreased creatinine clearance. The patient's hyperkalemia persisted while his serum creatinine began to normalize. Heparin was discontinued and replaced with fondaparinux $2.5 \mathrm{mg}$ subcutaneous. Upon this switch, the patient's potassium concentration began to normalize.

The implication of unfractionated heparin in this patient's hyperkalemia is supported secondary to chronological association, resolution upon alteration of therapy, and the inability to inculpate other interventions or baseline disease. Additionally, the persistent elevation of potassium in spite of repeated potassium-lowering interventions (i.e., insulin, albuterol, and furosemide) and the lowering of potassium in tube feedings add merit to this claim. A Naranjo Algorithm Adverse Drug Reaction Probability Score was tabulated at 6 , which indicates the reaction was probable. ${ }^{5}$ The only limitations to determine definite causality include failure to reintroduce the agent to the patient and a lack of monitoring serum drug concentrations or dosage adjustments, neither of which have values or recommendations suggested in the literature.

The appearance of hyperkalemia in our case implicates heparin as the inciting agent, as a significant increase in serum potassium occurred approximately four to seven days after its introduction, which is similar to that reported in the literature. ${ }^{6-8}$ Furthermore, the effect of acute kidney injury on our patient's degree of hyperkalemia is contradicted by the patient's improvements in serum creatinine over the same time frame (Figure 1).

Aldosterone synthesis is selectively reduced in patients treated with heparin, which may be associated with reduced number and affinity of adrenal angiotensin II receptors in the zona glomerulosa, which has been suggested to occur in as many as $7 \%$ of patients. ${ }^{6}$ Previous reports indicate that multiple factors are typically present that exacerbate the relative aldosterone deficiency and alter the ability of the host to compensate. This deficiency results in the reduced secretion of potassium into the lumen of the late distal tubule and collecting duct with subsequent hyperkalemia.

We switched the patient from enoxaparin to heparin 
as the patient's renal function began to deteriorate. The dosing of enoxaparin in patients with renal dysfunction is complex. A formula based on the patient's body weight, sex, and serum creatinine has been developed. ${ }^{9}$ We thought the simpler approach would be to switch the patient to heparin which has been used safely in trauma patients for decades. Also, we were uncertain when the patient's renal function would stabilize. If we continued using enoxaparin, we would have been continually adjusting the patient's dosing based on imprecise fluctuations in serum creatinine or repeated anti-Xa concentrations.

One must consider other possibilities for the patient's hyperkalemia. It is possible that this patient developed a contrast nephropathy. Those typically occur 24-48 hours after exposure to contrast dye. ${ }^{10}$ On arrival, as part of his trauma workup, this patient was exposed to contrast for multiple CT scans, but for the next several days, after those scans, the patient's creatinine actually decreased. The patient received $100 \mathrm{ml}$ of Isovue 370 on the day of admission as his only dose of intravenous contrast. The patient's creatinine was $1.4 \mathrm{mg} / \mathrm{dL}(0.70-1.40$ $\mathrm{mg} / \mathrm{dl}$ ) on admission. During the time in question, when the patient's creatinine begins to rise, the patient was not exposed to intravenous contrast dye within the previous 24-48 hours. This would eliminate the contrast dye as a possible cause of the patient's hyperkalemia.

Rhabdomyolysis is something else that needs to be considered. The patient had no clinical evidence of rhabdomyolysis. We did not obtain a creatinine kinase or a urine myoglobin while the patient was in the hospital. The patient had no evidence of compartment syndrome. Also, the patient had no evidence of adrenal dysfunction during this hospitalization. When the patient's creatinine began to rise as well as his potassium, a random cortisol level was obtained. It was $24.5 \mathrm{mcg} / \mathrm{dL}$ (3.1 $-22.4 \mathrm{mcg} / \mathrm{dl}$ ). This patient did not have persistent metabolic acidosis. The patient's past medical history of hypertension and diabetes predisposes him to renal dysfunction. This may have played a role in the patient's acute kidney injury as well as his hyperkalemia. The most likely cause of the patient's hyperkalemia is secondary to heparin. The timing of the rise in serum potassium and the fact that his potassium levels began to fall shortly after the discontinuing of the heparin is highly suggestive that heparin was the causative agent.

Finally, this trauma patient was hemodynamically unstable for his first six or seven days in the hospital. This is unusual. Most trauma patients will stabilize quickly once the source of the instability is found. We believe that the combination of the patient's spinal cord injury plus his community-acquired pneumonia caused the patient to vasodilate. Therefore, his systemic vascular resistance was low. We responded with fluids and vasoconstrictors which worked well. The patient's acute respiratory failure requiring APRV is confusing. The patient was somewhat overloaded, but diuresis didn't promptly improve the patient's pulmonary status. The patient was cultured several times, but we did not grow any new bacteria from the patient's lungs.

\section{CONCLUSION}

This case demonstrates the relevance of heparinassociated hyperkalemia and a need for the clinician to thoroughly consider this drug as a cause of refractory hyperkalemia in the critically ill patient. A prompt change to alternative agents like the factor Xa- inhibitors (i.e. fondaparinux) is an important step to resolve hyperkalemia in trauma patients who are at high risk for developing DVT.

\section{AUTHOR AFFILIATIONS}

1. Cabell Huntington Hospital, Huntington, West Virginia

2. Marshall University Joan C. Edwards School of Medicine, Huntington, West Virginia

\section{REFERENCES}

1. Geerts WH, Code KI, Jay RM, Chen E, Szalai JP. A prospective study of venous thromboembolism after major trauma. New England Journal of Medicine. 1994;331(24):1601-6.

2. Dennis JW, Menawat S, Von JT, Fallon JW, Vinsant GO, Laneve LM, Jagger C, Frykberg ER. Efficacy of deep venous thrombosis prophylaxis in trauma patients and identification of high-risk groups. 
The Journal of Trauma. 1993;35(1):132-8.

3. Britt LD, Zolfaghari D, Kennedy E, Pagel KJ, Minghini A. Incidence and prophylaxis of deep vein thrombosis in a high risk trauma population. The American Journal of Surgery. 1996;172(1):134.

4. Geerts WH, Jay RM, Code Kl, Chen E, Szalai JP, Saibil EA, Hamilton PA. A comparison of low-dose heparin with low-molecular-weight heparin as prophylaxis against venous thromboembolism after major trauma. New England Journal of Medicine. 1996;335(10):701-7.

5. Naranjo CA, Busto U, Sellers EM, Sandor P, Ruiz I, Roberts EA, Janecek E, Domecq C, Greenblatt DJ. A method for estimating the probability of adverse drug reactions. Clinical Pharmacology \& Therapeutics. 1981;30(2):239-45.

6. Abdel-Raheem MM, Potti A, Tadros S, Koka V, Hanekom D, Fraiman G, Danielson BD. Effect of low-molecular-weight heparin on potassium homeostasis. Pathophysiology of haemostasis and thrombosis. 2002;32(3):107-10.

7. Bengalorkar G, Sarala N, Venkatrathnamma P, Kumar T. Effect of heparin and low- molecular weight heparin on serum potassium and sodium levels. J Pharmacol Pharmacother. 2011; 2:266269.

8. Danguy C, Biston P, Carlier E, et al. Severe hyperkalemia in critically ill patients treated with prophylactic doses of enoxaparin. Intensive Care Med. 2012; 38:1904-1905.

9. Hulot JS, Vantelon C, Urien S, Bouzamondo A, Mahé I, Ankri A, Montalescot G, Lechat P. Effect of renal function on the pharmacokinetics of enoxaparin and consequences on dose adjustment. Therapeutic drug monitoring. 2004;26(3):305-10.

10. Murphy SW, Barrett BJ, Parfrey PS. Contrast nephropathy. Journal of the American Society of Nephrology. 2000;11(1):177-82. 